\title{
Aplicación de la técnica de componentes principales para el análisis de la pobreza en Honduras
}

Mirta Macías Ruano ${ }^{1}$

\section{RESUMEN}

El objetivo planteado en la presente investigación fue identificar los principales factores relacionados con la pobreza a partir de la aplicación del análisis de componentes principales (ACP), esta técnica de análisis exploratorio permite descubrir interrelaciones entre las variables y de acuerdo con los resultados, proponer los análisis estadísticos más apropiados. Adicionalmente, dicha técnica permite la reducción de un número considerable de variables en unos cuantos componentes que sintetizan las relaciones y variaciones del fenómeno analizado.

La metodología utilizada se caracteriza por ser cuantitativa, utilizando la información procedente de la encuesta permanente de hogares de propósito múltiple para los años 2001, 2005 y 2010, correspondientes al mes de septiembre, siendo el alcance correlacional con diseño transeccional no experimental.

Los resultados obtenidos a partir del ACP indican que la pobreza en Honduras no está caracterizada únicamente por factores de tipo económico, sino que cobran relevancia otros relacionados con la formación de capital humano, las características demográficas, el mercado de trabajo y las condiciones de habitabilidad de los hogares.

Palabras clave: análisis de componentes principales, pobreza e indicadores sintéticos.

1 Profesora universitaria, Carrera de Economía, Coordinadora Regional de Investigación UNAH-Valle de Sula: mirta.macias@unah.edu.hn 


\section{ABSTRACT}

The main goal in this research was to identify the main factors associated with poverty due to the application of principal component analysis (PCA). This exploratory analysis technique discovers the relationships between variables and according to the results, propose the most appropriate statistical analysis. In addition, this technique reduces considerably the number of variables into few components that synthesize the variations of the analyzed phenomenon.

The methodology used is characterized as quantitative, using data from the Permanent Survey on Multi purpose housing for the following: 2001, 2005 and 2010, during the month of September. The scope used can be defined as correlational with a non-experimental transactional design.

The ACP results indicate that poverty in Honduras is not only characterized by economic factors, but those related to human capital formation, demographic characteristics, the labor market and the living conditions of households.

Keyword: synthetic indicators, poverty and analysis of main components. 


\section{INTRODUCCIÓN}

Honduras es uno de los países más pobres de América Latina, no obstante, los estudios al respecto son limitados y basados en metodología y medición tradicional (líneas de pobreza y necesidades básicas insatisfechas que describen tasa de incidencia). Bajo tal escenario, surge el interés por estudiar dicho fenómeno usando un abordaje metodológico de carácter multidimensional que permita identificar factores (multicausales) que influyen en las condiciones de pobreza en los hogares hondureños.

Dada la realidad social hondureña, la Universidad Nacional Autónoma de Honduras prioriza dicho tema en el Eje de investigación No.1: Desarrollo económico y social; concretamente, en el Tema prioritario No. 2: Pobreza e inequidad. En lo que respecta a los resultados de la investigación, se aporta una nueva perspectiva de análisis y medición de pobreza y a la vez se contribuye a la reducción de la brecha de conocimiento en un problema de relevancia social por las altas tasas de incidencia que presentan los hogares hondureños.

Finalmente, hay que mencionar que las limitaciones para el desarrollo de la investigación fueron mínimas y se superaron con la revisión de las tres bases de datos, en las cuales se identificaron inconsistencias en ciertas variables, por ello se homogenizaron los criterios de selección en las variables utilizadas para los modelos del ACP utilizados.

\section{METODOLOGÍA}

Cuando se estudia un fenómeno tan complejo como la pobreza, se encuentra el dilema teórico o metodológico sobre qué variables deben ser consideradas, de tal manera que su explicación incluya aspectos económicos y sociales, entre otros, y que cuenten con una sustentación teórica. Ante esta circunstancia, el análisis de la pobreza mediante métodos cuantitativos que ayuden a agrupar poblaciones a partir de características similares, auxilian al investigador a resumir grandes cantidades de variables que pueden estar correlacionadas en pocos componentes que lo simplifiquen.

En tal contexto, el enfoque utilizado en la investigación es cuantitativo, debido a que 
se utilizó información sociodemográfica, educativa y económica de la encuesta permanente de hogares de propósitos múltiples del mes de septiembre para los años 2001, 2005 y 2010 (siendo transeccional) que realiza el Instituto Nacional de Estadística, lo que permitió obtener resultados que permitieron identificar y medir la pobreza en los hogares hondureños.

El alcance del estudio en un primer momento es descriptivo, porque se buscaba caracterizar los hogares hondureños en situación de pobreza o no pobreza, para posteriormente examinar la relación existente entre las variables analizadas y el nivel o intensidad de dicha relación mediante un análisis de correlación.

El diseño de la investigación es no experimental, porque no se pretendía manipular variables, únicamente analizar la pobreza para obtener los resultados que se querían a partir de los objetivos propuestos y dar respuestas a las preguntas investigación.

La unidad de análisis está constituida por los hogares principales como criterio de inclusión, por tal motivo se excluye segundo o más hogares que pudieran habitar en una misma vivienda. Los hogares se seleccionaron aplicando la opción "select case" para hogar igual 1 en el programa SPSS 18. La distribución del tamaño muestral se presenta en el cuadro 1.

Cuadro 1. Total hogares usados para ACP

No. De Hogares

16237

7195

8828

\section{Años}

2001

2005

2010

Fuente: elaboración propia con base a EHPPM, INE, 2001, 2005 y 2010.

Definida la unidad de análisis, el siguiente paso consistió en seleccionar las variables que serían analizadas en los diferentes modelos utilizados, aplicando la técnica estadística de análisis de componentes principales (ACP).

Tal proceso consistió en realizar combinaciones con las variables seleccionadas (52), a fin de obtener un modelo explicativo que resuma las condiciones de pobreza desde la perspectiva estadística y teórica en el período de referencia, finalmente se obtuvo un modelo a partir de 18 variables de caracterización de los hogares para cada año (ver anexos). Vale mencionar que toda la información de las tres bases de datos se procesó con el paquete estadístico SPS 18 (ver cuadro 2). 
Cuadro 2. Variables usadas para modelo ACP 2001, 2005 y 2010

\begin{tabular}{|c|c|c|c|}
\hline No. & Etiqueta & Nombre de la variable & Fuente \\
\hline 1 & Ingtrabajo & Ingreso del trabajo & INE \\
\hline 2 & Ocupadosporc & Ocupados & INE \\
\hline 3 & Horastrab1 & Jornada laboral ocupación principal & INE \\
\hline 4 & Remesas & Remesas & INE \\
\hline 5 & Esolarjefe & Escolaridad del jefe del hogar & INE \\
\hline 6 & Ingperca & Ingreso per cápita & INE \\
\hline 7 & Materpiso & Material del piso & INE \\
\hline 8 & Materpared & Material de la pared & INE \\
\hline 9 & Saneamiento & Saneamiento & INE \\
\hline 10 & Menor15por & Menores de 15 años & INE \\
\hline 11 & Dependem & Dependencia demográfica & INE \\
\hline 12 & Hacinam & Hacinamiento & INE \\
\hline 13 & Tamañoh & Tamaño del hogar & INE \\
\hline 14 & Ocupadotrab2 & Ocupados con segundo empleo & INE \\
\hline 15 & Peagricola & Ocupados sector primario (\%) & INE \\
\hline 16 & Peaservicios & Ocupados sector servicios (\%) & INE \\
\hline 17 & Depecon & Dependencia económica & INE \\
\hline 18 & Inactivos & Población inactiva (\%) & INE \\
\hline
\end{tabular}

Fuente: elaboración propia a partir de ACP 2001, 2005 y 2010.

Figura 1. Modelo linealXP

$$
\begin{aligned}
& X_{1}=\mathbf{a}_{11} F_{1}+\mathbf{a}_{12} F_{2}+\ldots+\mathbf{a}_{11} F_{j}+\ldots+\mathbf{a}_{1 \mathrm{~m}} F_{\mathrm{m}}+\mathbf{d}_{1} \mathbf{U}_{1} \\
& \mathbf{X}_{\mathrm{i}}=\mathbf{a}_{\mathrm{i} 1} F_{1}+\mathbf{a}_{\mathrm{i} 2} F_{2}+\ldots+\mathbf{a}_{\mathrm{ij}} F_{\mathrm{j}}+\ldots+\mathbf{a}_{\mathrm{im}} F_{\mathrm{m}}+\mathbf{d}_{\mathrm{i}} \mathbf{U}_{\mathrm{i}} \\
& \mathbf{X}_{\mathrm{p}}=\mathbf{a}_{\mathrm{p} 1} F_{1}+\mathbf{a}_{\mathrm{p} 2} F_{2}+\ldots+\mathbf{a}_{\mathrm{p}} F_{\mathrm{j}}+\ldots+\mathbf{a}_{\mathrm{pm}} F_{\mathrm{m}}+\mathbf{d}_{\mathrm{p}} \mathbf{U}_{\mathrm{p}}
\end{aligned}
$$


Como ya se ha indicado antes, uno de los métodos más utilizados en este tipo de estudios es el ACP, este trata de explicar $p$ variables observables $\mathrm{X} 1, \mathrm{X} 2, \ldots, \mathrm{Xp}$ por $p+m(m<p)$ variables incorrelacionadas no observables F1, F2, .., Fm, U1, U2, .., Up que denominaremos factores únicos, de acuerdo con modelo lineal que se observa en la figura 1.

La matriz de datos $(X)$ se expresa de la siguiente manera: $X=A^{*} F+D$; donde $A$ es una matriz de patrón factorial que recoge la relación entre las variables y los factores comunes y $\mathrm{D}$ es una matriz que recoge los coeficientes de los factores únicos.

Para evaluar el modelo factorial se utilizaron tres contrastes: matriz de correlación, test de esfericidad de Barlet y medida de la adecuación muestral Kaiser, Meyer y Olkin (KMO); los criterios de selección de los factores considerados fueron: valores propios mayores que 1 , el porcentaje de la varianza total explicada y el gráfico de sedimentación.

El procedimiento de rotación de factores correspondiente a este trabajo es el varimax, el cual permite que los ejes rotados se obtengan a partir de maximizar la suma de la cargas factoriales al cuadrado dentro de cada factor y una de sus propiedades más importantes es que después de aplicada la varianza total explicada por los factores y la comunalidad de cada variable no se ve modificada, aunque por supuesto, sí cambia el porcentaje de varianza explicada por cada eje.

\section{RESULTADOS}

Para la interpretación de los resultados se precisa observar la correlación entre las variables originales y el subconjunto de variables agrupadas en componentes, porque no existe una estructura tipo que permita comprender claramente los componentes, debido a que los mismos no se obtienen automáticamente.

Así, los resultados señalan que la agrupación obtenida a partir del ACP para los distintos años analizados tienen una interpretación adecuada recogiendo cada uno de los componentes variables homogéneos y que en conjunto explican más de un 64 $\%$ de la varianza, porcentaje que se considera adecuado (Herrero y otros, 2006) y se expresan de la siguiente manera: 
1. Cuatro componentes (condiciones de habitabilidad, ingresos y ocupación, inserción laboral y dependencia demográfica) corresponden con la estructura factorial del año 2005 y 2010.

2. El factor demográfico se recoge en dos componentes; por un lado, la dependencia demográfica y los menores de 15 años que se representan en el componente 3; y por otra parte, el tamaño del hogar, el hacinamiento y en menor medida los menores de 15 años sintetizados en el factor 6 .

3. Los componentes son comparables entre los años 2001, 2005 y 2010, dado que aluden a las mismas características de los hogares hondureños.

\section{Modelo ACP 2010}

En el modelo del año 2010, se obtienen cinco componentes que definen la situación de los hogares analizados, con una varianza explicativa de un $64.14 \%$ (ver cuadro 3). El primer factor (F1) se denomina demográfico y explica aproximadamente el 24.167 $\%$ de la varianza y una asociación directa entre las variables para este factor.

El segundo factor (F2) se nombró como condiciones de habitabilidad y saneamiento y recoge aproximadamente un $16.663 \%$ de la varianza y las correlaciones son positivas en todas las variables referidas a la calidad de los materiales de la infraestructura de la vivienda y el acceso a los servicios básicos; así, los hogares con mayores puntuaciones en este eje serán los que tienen mejores condiciones de habitabilidad.

El factor (F3) denominado fuerza laboral e ingresos, engloba la información de las variables relacionadas con los ingresos y la dotación de recurso humano. Explica alrededor de un $9.935 \%$ de la varianza y presenta altas correlaciones con signo positivo para las variables: ocupados e ingresos por trabajo y negativa con los desempleados.

El cuarto factor $(F 4 \bullet)$ nombrado educación, explica el $6.815 \%$ de la variabilidad y comprende información del total de años de educación aprobados por el jefe del hogar y de población sin acceso a educación en el hogar. La asociación entre las variables es inversa, es decir, que el acceso y el logro educativo de la población en edad escolar se asocia al nivel educativo del jefe o jefa de hogar, por ello, el menor nivel educativo en la jefatura del hogar se asocia al problema de alta población sin acceso a educación. 
El quinto factor (F5), se llama inserción laboral, explica alrededor del $6.594 \%$ de la variabilidad y comprende información de las personas ocupadas según el sector de inserción laboral y con segundo empleo. Se identifica una correlación alta y positiva con los ocupados en el sector servicios y negativa para inserción laboral de los servicios (ver cuadro 3).

Cuadro 3. Resultado del análisis de componentes principales 2010

\begin{tabular}{llllll}
\hline & \multicolumn{5}{c}{ Factores } \\
Variable & F1 & F2 & F3 & F4 & F5 \\
\hline Dependencia económica & 0.883 & -0.002 & -0.048 & -0.030 & 0.111 \\
\hline Dependencia demográfica & 0.878 & 0.002 & -0.059 & -0.034 & 0.113 \\
\hline Tamaño del hogar & 0.869 & -0.024 & -0.136 & 0.099 & 0.015 \\
\hline Hacinamiento & 0.769 & 0.144 & -0.030 & 0.147 & 0.000 \\
\hline Material piso & 0.058 & 0.746 & 0.114 & 0.163 & 0.046 \\
\hline Material pared & 0.060 & 0.658 & 0.099 & 0.097 & 0.007 \\
\hline Ocupados en servicios & 0.450 & -0.553 & 0.151 & 0.094 & -0.485 \\
\hline Saneamiento & 0.032 & 0.497 & 0.016 & 0.132 & 0.019 \\
\hline Inactivos & 0.105 & -0.136 & -0.832 & 0.111 & 0.002 \\
\hline Ocupados & -0.419 & 0.205 & 0.678 & -0.067 & 0.075 \\
\hline Ingreso por trabajo & 0.261 & -0.496 & 0.560 & 0.253 & 0.104 \\
\hline Población sin educación & 0.121 & 0.132 & -0.067 & 0.843 & 0.131 \\
\hline Escolaridad del jefe & -0.001 & -0.457 & 0.024 & -0.674 & -0.032 \\
\hline Subempleo & 0.203 & 0.063 & 0.232 & 0.046 & 0.701 \\
\hline Ocupados agricultura & 0.466 & 0.327 & -0.017 & 0.087 & 0.652 \\
\hline Remesas & -0.140 & -0.248 & -0.109 & 0.132 & 0.385 \\
\hline
\end{tabular}

Varianza explicada (\%)

\begin{tabular}{lll} 
F1 & Demográficos y mercado laboral & 24.167 \\
F2 & Condiciones de habitabilidad y saneamiento & 16.663 \\
F3 & Fuerza laboral e ingreso & 9.935 \\
\hline
\end{tabular}


Varianza explicada (\%)

F4

F5

Varianza explicada
Educación

Inserción laboral
6.815

6.594

64.143

Método de extracción: análisis de componentes principales

Método de rotación: normalización de varimax con káiser

Fuente: elaboración propia en base a resultados ACP 2010.

\section{Modelo ACP 2005}

En el año 2005, el ACP ha resumido la información original en un total de cinco, con una varianza explicada de un $65.8 \%$ para el modelo. El primer factor (F1) llamado condiciones de habitabilidad y servicios y explica aproximadamente el $17.2 \%$ de la varianza y una asociación directa entre todas variables.

El factor (F2) se denomina ingresos y ocupación, engloba variables relacionadas con los ingresos, personas ocupadas, la jornada laboral de la primera ocupación en los hogares y explica aproximadamente un $15.9 \%$ del total de la varianza. Presenta altas correlaciones con signo positivo con todas las variables, lo que supone que a mayor proporción de personas ocupadas por hogar, mayor ingreso.

El tercer factor (F3), de nombre demográfico, mide la dinámica poblacional a través de variables como la estructura de edad, tamaño del hogar, la dependencia demográfica, el grado de hacinamiento y recoge aproximadamente el $13.9 \%$ de la varianza explicada; su correlación indica que hay relación directa entre las variables analizadas.

El cuarto factor (F4) es la dependencia económica. Explica aproximadamente el 11.6 $\%$ de la varianza y resume información de la población ocupada y de la que está inactiva; su correlación es positiva, por lo que confronta a los hogares con mayor relación de dependencia económica con los que tienen un menor valor en dicho eje.

El quinto factor (F5), llamado capacidad de subsistencia, explica alrededor del $8.5 \%$ de la variabilidad y comprende información del total de años de educación aprobados por el jefe del hogar, así como del ingreso per cápita. La correlación es positiva por lo que la educación del jefe e ingresos están directamente relacionadas (ver cuadro 4). 
Cuadro 4. Resultados del análisis de componentes principales 2005

\begin{tabular}{|c|c|c|c|c|c|}
\hline \multirow[b]{2}{*}{ Variable } & \multicolumn{4}{|c|}{ Factores } & \multirow[b]{2}{*}{ F5 } \\
\hline & F1 & F2 & F3 & F4 & \\
\hline Calidad de la pared & 0.765 & -0.017 & -0.017 & -0.051 & 0.215 \\
\hline Calidad del piso & 0.723 & 0.062 & 0.062 & -0.141 & 0.280 \\
\hline Calidad del saneamiento & 0.709 & 0.014 & 0.014 & -0.122 & 0.291 \\
\hline Ocupado en la agricultura & -0.674 & 0.158 & 0.158 & 0.003 & -0.135 \\
\hline Subempleo & -0.633 & 0.245 & 0.245 & 0.070 & 0.301 \\
\hline Ocupados en los servicios & 0.604 & -0.042 & -0.042 & -0.084 & -0.124 \\
\hline Ocupados & -0.111 & 0.160 & 0.160 & -0.010 & 0.112 \\
\hline Horas empleo principal & 0.165 & 0.107 & 0.107 & -0.059 & -0.042 \\
\hline Ingreso por trabajo & -0.006 & -0.036 & -0.036 & 0.030 & 0.020 \\
\hline Ingresos por remesas & 0.111 & -0.629 & 0.108 & 0.081 & 0.048 \\
\hline Inactivos & -0.041 & -0.178 & 0.918 & 0.050 & -0.163 \\
\hline Tasa de dependencia económica & -0.115 & 0.108 & 0.853 & 0.048 & 0.067 \\
\hline Tamaño del hogar & -0.051 & 0.153 & 0.698 & 0.407 & -0.317 \\
\hline Menor de 15 años & -0.072 & -0.007 & 0.126 & 0.910 & -0.092 \\
\hline Tasa de dependencia demográfica & -0.110 & -0.221 & 0.033 & 0.853 & -0.053 \\
\hline Tasa de hacinamiento & -0.299 & 0.153 & 0.355 & 0.520 & -0.295 \\
\hline Ingreso per cápita & 0.168 & 0.030 & -0.042 & -0.071 & 0.720 \\
\hline Años educación aprobados jefe & 0.270 & -0.010 & -0.260 & -0.194 & 0.666 \\
\hline \multicolumn{6}{|l|}{ Varianza explicada (\%) } \\
\hline $\mathbf{F 1}$ & \multicolumn{4}{|c|}{ Condición habitabilidad y tipo inserción laboral } & $17.2 \%$ \\
\hline $\mathbf{F 2}$ & \multicolumn{4}{|c|}{ Fuentes de ingresos } & $14.9 \%$ \\
\hline F3 & \multicolumn{4}{|c|}{ Dependencia económica } & $13.3 \%$ \\
\hline F4 & \multicolumn{4}{|c|}{ Dependencia demográfica } & $11.6 \%$ \\
\hline F5 & \multicolumn{4}{|c|}{ Capacidad de subsistencia } & $8.5 \%$ \\
\hline \multicolumn{5}{|l|}{ Total varianza explicada } & $65.8 \%$ \\
\hline
\end{tabular}

Fuente: elaboración propia con base a resultados ACP 2005. 


\section{Modelo ACP 2001}

Finalmente, en el año 2001, se obtienen seis componentes, tal como se describe en el cuadro 5, con una varianza explicada del $64.296 \%$. El primer factor (F1) se llama factor condiciones de habitabilidad. Explica el $13.9 \%$ de la varianza y sintetiza la información de las condiciones de la infraestructura de las viviendas, como es el caso de los materiales de paredes y pisos. Además, incluye el acceso a servicios básicos. Las variables en su mayoría están altamente correlacionadas con el componente y de manera positiva, por tanto, valores altos del indicador indican una situación más privilegiada en cuanto a las condiciones de habitabilidad.

El segundo factor (F2), llamado factor ingresos y condición de actividad, explica un 11. $865 \%$ de la variabilidad y resume la información a nivel del hogar en cuanto al porcentaje de personas ocupadas, los ingresos por trabajo, las remesas y en menor proporción el porcentaje de inactivos. Se identifica una correlación positiva con las variables ocupados e ingresos por trabajo y negativa con las remesas y los inactivos, de esta forma se contrapone a los hogares que tienen mayores ingresos más ocupados con los que tienen ingresos por remesas y más inactivos.

El tercer factor (F3), designado estructura del hogar, tiene una varianza de $11.438 \%$ y la relación entre las variables es positiva, por lo que sugiere que hogares con mayores puntuaciones son los que tienen una composición familiar grande y mayor hacinamiento.

El cuarto factor (F4) es claramente identificable e incorpora variables como el porcentaje de inactivos, la relación de dependencia económica, ocupados en el sector servicios y en menor proporción los ocupados con un segundo empleo; por tal motivo, lo llamaremos dependencia económica, explica el $9.69 \%$ de la variabilidad, todas las variables están relacionadas de manera positiva.

El quinto factor (F5), denominado inserción laboral y nivel educativo, resume un $8.7 \%$ de la variabilidad proporcionando información sobre el vínculo entre educación (años de estudios aprobados) y la inserción laboral (población ocupada tanto en el sector servicios y como en el sector agrícola). Se identifica una relación inversa entre los que laboran en el sector primario y los de un sector moderno, lo cual podría ser indicativo de la contraposición de la actividad económica a partir de la geografía, urbano versus rural. La variable escolaridad del jefe presenta una relación positiva.

Finalmente, el sexto factor (F6) es de fácil comprensión, ya que explica un $8.54 \%$ de 
la variación y recoge información sobre el porcentaje de población menor de 15 años y la relación de dependencia demográfica. Este factor tiene una correlación positiva, por ello los valores altos del indicador sugieren hogares con mayor proporción de jóvenes (menores de 15 años) y mayor dependencia demográfica (ver cuadro 5).

Cuadro 5. Resultados ACP 2001

\begin{tabular}{lcccccc}
\hline & \multicolumn{7}{c}{ Factores } \\
\hline Variable & F1 & F2 & F3 & F4 & F5 & F6 \\
\hline Calidad material del piso & $0 ., 810$ & 0.013 & -0.057 & -0.063 & 0.125 & -0.088 \\
\hline Saneamiento & 0.768 & 0.024 & -0.067 & -0.130 & 0.206 & -0.102 \\
\hline Calidad material de la pared & 0.759 & 0.002 & -0.020 & 0.010 & -0.042 & -0.010 \\
\hline Acceso a agua & 0.689 & -0.012 & 0.015 & -0.034 & 0.034 & 0.015 \\
\hline Ingreso por trabajo & 0.042 & 0.829 & 0.000 & 0.205 & 0.105 & 0.025 \\
\hline Ocupados & 0.008 & 0.748 & 0.008 & 0.374 & 0.121 & 0.019 \\
\hline Remesas & 0.011 & -0.637 & -0.013 & 0.024 & 0.034 & 0.001 \\
\hline Inactivos & 0.016 & -0.568 & 0.029 & 0.503 & 0.130 & 0.089 \\
\hline Tamaño del hogar & -0.039 & -0.003 & 0.924 & 0.050 & 0.003 & -0.088 \\
\hline Hacinamiento & -0.079 & 0.013 & 0.905 & 0.010 & -0.176 & 0.043 \\
\hline Dependencia económica & -0.009 & 0.138 & -0.056 & 0.747 & -0.015 & -0.122 \\
\hline Ocupados sector agrícola & -0.118 & 0.062 & -0.026 & 0.570 & -0.535 & -0.120 \\
\hline Subempleo & -0.115 & 0.110 & 0.092 & 0.473 & -0.011 & 0.136 \\
\hline Ocupados sector servicios & 0.034 & 0.086 & -0.087 & 0.204 & 0.759 & -0.102 \\
\hline Años aprobados jefe de hogar & 0.173 & 0.012 & -0.067 & -0.186 & 0.695 & -0.023 \\
\hline Dependencia demográfica & -0.087 & -0.003 & 0.140 & -0.033 & -0.029 & 0.901 \\
\hline Menores 15 años & -0.075 & 0.003 & -0.466 & 0.064 & -0.083 & 0.738 \\
\hline
\end{tabular}




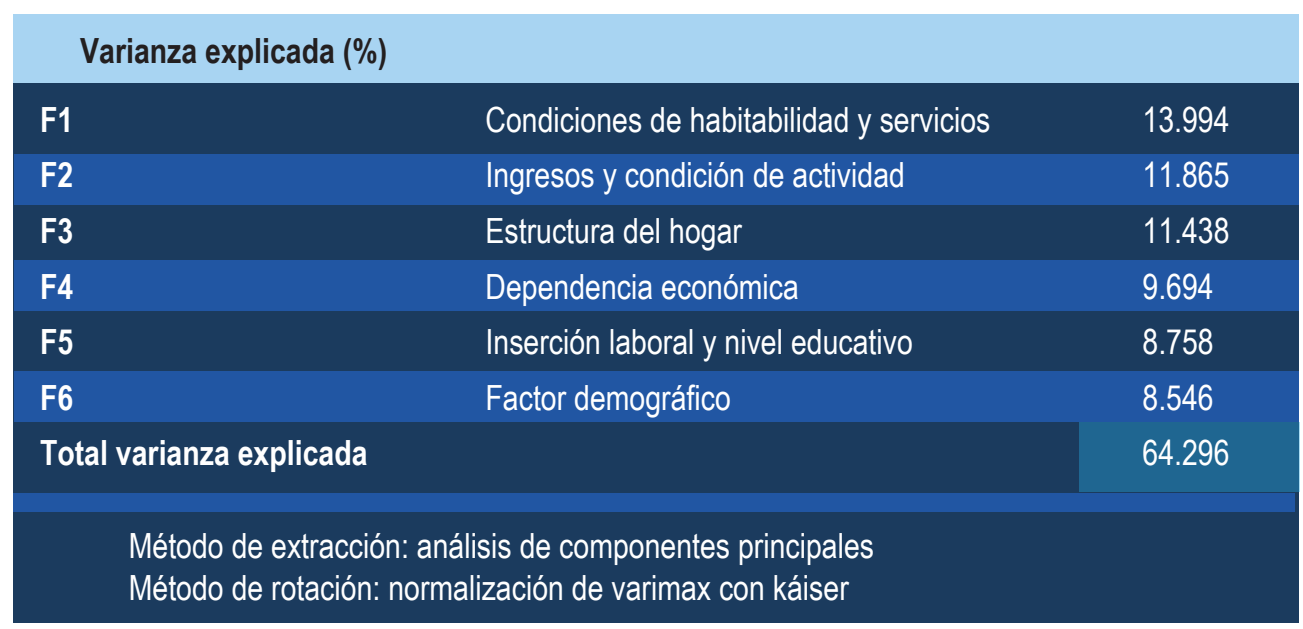

Fuente: elaboración propia con base a resultados ACP 2001.

\section{DISCUSIÓN DE RESULTADOS}

El propósito de esta investigación fue obtener los factores que explican la pobreza, los mismos han sido sintetizados en componentes que tienen una sustentación teórica y desde la perspectiva estadística las correlaciones obtenidas son las esperadas en función de la teoría.

Los resultados obtenidos a la luz de la teoría y le evidencia empírica en la investigación, permiten deducir que la pobreza en los hogares hondureños está asociada a factores demográficos, condiciones de habitabilidad y saneamiento, así como a aspectos relacionados con el mercado laboral, los ingresos y aspectos educativos.

Lo antes expuesto sugiere que el fenómeno analizado es el resultado de una compleja situación que enfrentan los hogares hondureños, en donde los elementos demográficos se asocian a factores educativos, estos, a su vez, con las condiciones de habitabilidad y saneamiento. De igual forma, el mercado laboral, su forma de inserción y la remuneración también inciden en las condiciones de los hogares, así como las remesas. Todo ello ha sido evidenciado desde la perspectiva teórica, igualmente que la unión de estos factores determinan las condiciones de pobreza en los hogares. 


\section{CONCLUSIONES}

Los factores que inciden en la pobreza de los hogares hondureños, según los resultados obtenidos a partir del ACP, indican que en Honduras no está caracterizada únicamente por factores de tipo económico, sino que cobran relevancia otros relacionados con la formación de capital humano, las características demográficas, el mercado de trabajo y las condiciones de habitabilidad de los hogares.

De forma general, en los tres años analizados, los factores identificados recogen las condiciones de habitabilidad de las viviendas, las fuentes de ingresos, el tipo de inserción laboral de los miembros del hogar, su estructura demográfica y su dependencia y capacidad económica. Estos factores son coherentes con los principales enfoques de pobreza.

\section{AGRADECIMIENTO}

A la Dirección de Investigación Científica y Posgrado por la oportunidad de publicar este artículo.

\section{BIBLIOGRAFÍA}

Adams, R.H. (2006). Economic Growth, Inequality and Poverty: The Estimating the Growth Elasticity of Poverty. World Development, 32(12), 1989-2014. Recuperado de: http/:www.elsevier.co,/locate /worlddev.

Aguilar, M. J. y Ander-Egg, E. (1992). Evaluación de servicios y programas sociales. Madrid: Siglo XXI Editores.

Aguirre, H.; Calderón, M. y Wlasiuk, J. (2003). Desempeño de los indicadores socioeconómicos seleccionados en Argentina, 1980-2001. Documento de trabajo 2003-2005. Fundación Pent. Recuperado de: http/ www.pentfundación.or/pent_2003-2005.pdf.

Aguirre Tobón, K. (2004): Convergencia en estándares de vida en Colombia (1985-2000). Enfoque no paramétrico. Centro de Recursos para el Análisis de Conflictos. Recuperado de: htpp/www.cerac.org.co. 
Albi, E.; Gonzales Paramo, J. y Zubiri, I. (2000). Economía pública I. Barcelona: Ariel. Banco Mundial. (2006). Reducción de la pobreza y crecimiento: círculos virtuosos y círculos viciosos. Washington D.C.

Banco Mundial. (2001). Informe de desarrollo mundial. Washington D.C.

Banco Mundial. (2000). Informe de desarrollo mundial. Washington D.C.

Boltvinik, J. (2003). Tipología de los métodos de medición de la pobreza. Los métodos combinados. Revista de Comercio Exterior, 53 (5), 453-465.

Comisión Económica para América Latina. (2004). Pobreza y vulnerabilidad social:

Mercado de trabajo e inversión social en el istmo centroamericano a inicios del milenio. Santiago de Chile: Naciones Unidas.

Dieterlen, P. (2003). La pobreza: un desafío filosófico. México D.F: Universidad Nacional Autónoma de México.

Fernández Santana, J.O. (1998). Comprensión y manejo del análisis factorial. Revista Internacional de Sociología, 46, 7-35.

Herrero, L.C.; Figueroa, V. y Sanz, J. (2006). Las disparidades territoriales en Castilla y León: Estudio de la convergencia económica a nivel municipal. Revista de Investigación económica y Social de Castilla y León, 9, 15-170.

Organización Internacional del Trabajo. (2000). Panorama Laboral de América Latina y el Caribe. Ginebra. 